\section{Pancreatic metastasis of a meningeal hemangio- pericytoma: a rare cause of obstructive jaundice}
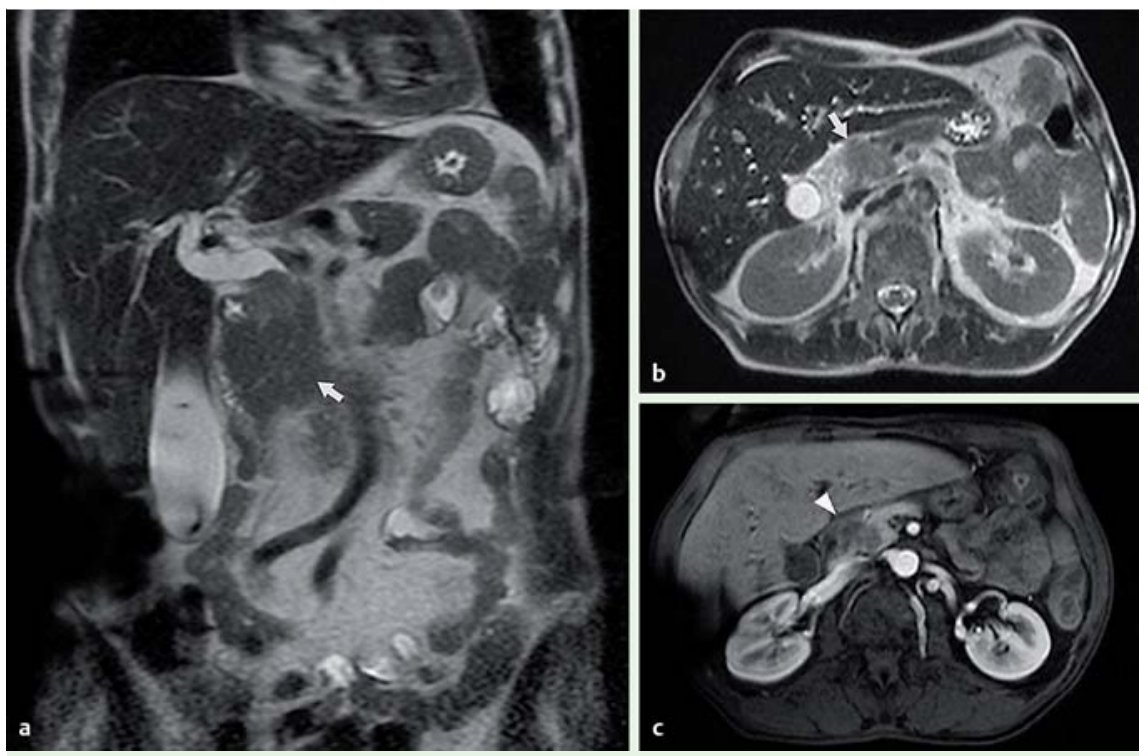

Fig. 1 Magnetic resonance imaging (MRI) in a 52-year-old man with obstructive jaundice and a known meningeal hemangiopericytoma showing biliary duct dilatation, an enlarged gallbladder and a heterogeneous mass in the pancreatic head, which appeared: a, b hypointense on T2-weighted fast-spin echo images (arrows) in coronal and axial views; $\mathbf{c}$ hypovascular during the arterial phase of a contrast-enhanced T1-weighted gradient echo image (arrowhead).

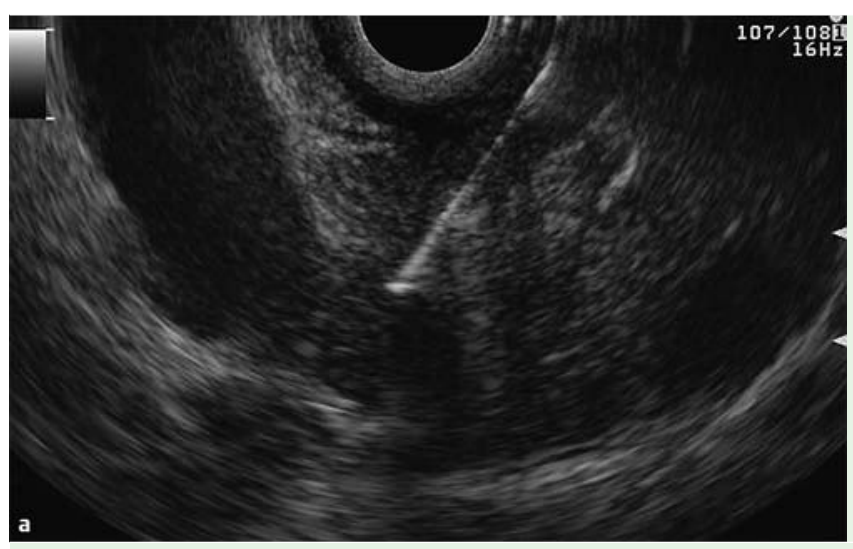

Fig. 2 Endoscopic ultrasound (EUS) images showing: a a $21-\mathrm{mm}$ hypoechoic heterogeneous pancreatic head mass which is being sampled by fine needle aspiration (FNA) using a $25 \mathrm{C}$ needle; b a smaller $14-\mathrm{mm}$ hypoechoic mass in the uncinate process close to the superior mesenteric vein.
A 52-year-old man presented to the emergency department with jaundice, pale stools, and dark urine for 1 week, having lost $15 \%$ of his body weight during the previous month. He was a heavy smoker and drinker. He had been diagnosed 4 months previously with a meningeal hemangiopericytoma that had been surgically resected then treated with cranial external radiotherapy. Laboratory evaluation was remarkable for hyperbilirubinemia $(9 \mathrm{mg}$ / $\mathrm{dL}$ ) and cholestasis (alkaline phosphatase [ALP] $1300 \mathrm{IU} / \mathrm{L})$. Magnetic resonance imaging (MRI) revealed a 25-mm pancreatic head mass and upstream dilatation of the bile ducts but a normal main pancreatic duct ( Fig. 1). A second 15-mm mass in the uncinate process was also noted.

The patient underwent an endoscopic ultrasound (EUS) using a linear echoendoscope (UCT 10-140 AL5; Olympus), which revealed a slightly heterogeneous, hypoechoic, ill-defined mass in the pancreatic head from which a fine needle aspiration (FNA) was taken with a 25G needle (Wilson Cook; Fig.2a). The second smaller lesion was also visualized in the uncinate process close to the superior mesenteric vein ( Fig. 2 b).

During the same session, endoscopic retrograde cholangiopancreatography (ERCP) was performed as a tandem procedure and a 7-cm long, 7-Fr plastic biliary prosthesis was placed to allow adequate biliary drainage. Evaluation of the cell block cytology was consistent with hemangiopericytoma ( $\bullet$ Fig.3). The patient died 2 weeks later from uncontrolled seizures.

Endoscopy_UCTN_Code_CCL_1AF_2AZ_3AB

Competing interests: None

Lídia Roque Ramos ${ }^{1}$, Pedro Pinto Marques $^{1}$, Rui Loureiro ${ }^{1}$, Maria José Brito ${ }^{2}$, João de Freitas ${ }^{1}$

${ }^{1}$ Gastroenterology Department, Hospital Garcia de Orta, Almada, Portugal 2 Pathology Department, Hospital Garcia de Orta, Almada, Portugal 


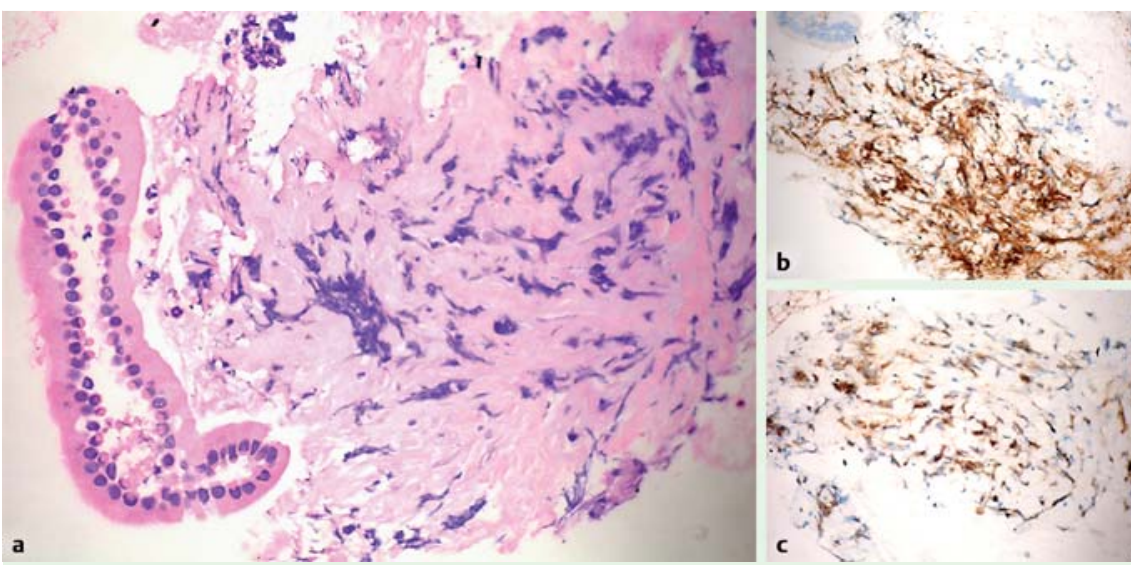

Fig. 3 Cytology examination of the pancreatic mass showing: a numerous crowded atypical cells with pleomorphic nuclei (hematoxylin and eosin [H\&E] stain; magnification $\times 300$ ); b positive staining for CD34; c positive staining for bcl-2.
Bibliography

Dol http://dx.doi.org/

10.1055/s-0033-1359158

Endoscopy 2014; 46: E135-E136

(c) Georg Thieme Verlag KG

Stuttgart · New York

ISSN 0013-726X

\section{Corresponding author}

\section{Lídia Roque Ramos, MD}

Gastroenterology Department

Hospital Garcia de Orta

Avenida Torrado da Silva

2801-951 Almada

Portugal

Fax: +351-212-957004

lidia.roque.ramos@gmail.com 